\title{
Power Spectrum Estimation for Narrowband and Broadband Applications using Subspace Method
}

\author{
Poonam Yadav \\ ME Scholar \\ ECE Deptt. NITTTR \\ Chandigarh, India
}

\author{
Rajesh Mehra \\ Associate Professor \\ ECE Deptt.NITTTR \\ Chandigarh, India
}

\begin{abstract}
This paper reveals the comparison of various estimation methods to evaluate the most suitable among parametric, nonparametric and subspace method for narrowband and broadband applications using MATLAB. Comparison is done on the basis of same SNR, Overlay and complex sinusoid. Subspace method proved to be the best as the Estimated PSD is in close proximity with True PSD. Also, reduced spectral leakage of $7.5 \%$ and side lobe attenuation of $-11.3 \mathrm{~dB}$ has been achieved by using Music and Eigen vector in subspace method whereas in non parametric method spectral leakage is $9.12 \%$ and side lobe attenuation is $-13.2 \mathrm{~dB}$ with noise in the signal.
\end{abstract}

\section{Keywords}

PSD, SNR, Spectral Leakage, Side lobe attenuation.

\section{INTRODUCTION}

The energy distribution of a time sequence in the frequency content is characterised by power spectrum. Energy is real entity, so the power spectrum does not provide information about phase. As a time sequence may contain non-periodic or sampled periodic signal components, the power spectrum of a time sequence is taken to be a continuous function of frequency. When sequence of sampled frequency to represent the continuous frequency is taken, then the frequency interval is proportional to this specific frequency. To remove this dependency on interval, power spectrum can be normalised to have the spectral analysis, which is the ratio of power spectrum to frequency interval. In this method the Rectangular window is used that has good resolution for sinusoids of compatible strength. This window provides less spectral leakage in subspace method with low side lobe attenuation. It plays vital role in establishing frequency response of FIR filter. In signal processing, FIR filter is a filter whose response to any finite length input is of finite duration because it settles to zero in finite time [1].

The undesirable effects are reduced by the use of window that does not contain swift discontinuities in time domain characteristics. Both Rectangular and Hamming window with identical $\mathrm{M}$, the main lobe width is even wider for these windows in comparison with other windows [2]. PSD estimation methods are as follows:

Parametric methods: These methods depends on parametric models of a time sequence, such as AR, MA and ARMA model and thus called model-based methods. Estimation of PSD of a time sequence with model based method requires the model parameters of the time series at the beginning. AR model is widely used as it represents the spectra with narrow peaks [3]. Design of an appropriate model that perfectly reflects the behaviour of the system is to be taken care of otherwise the estimated PSD might not be good in quality or performance.
Non parametric methods: The periodogram, Welch method, Thompson Multitaper and Capon method, are based on DFT where time series parameters are need not to be obtained. But they have implementation possible using FFT. But in case of short data lengths they have drawback of limited frequency resolution. Also, care is to be taken to obtain meaningful result. On the other hand parametric methods provide high resolution along with computational efficiency [4].

Subspace methods: The multiple signal classification method (MUSIC) and Eigen Vector method also are model based method as parametric ones. The main drawback of nonparametric methods is that the computation produces distortion in the resultant densities. The key merit of non parametric methods is the wholesomeness which means the estimated power spectral density does not contain spurious frequency peaks. In contrast, data windowing is not used in parametric methods. Parametric methods assume that a signal fits a particular model. The estimated PSDs may contain specious frequency peaks if the assumed model is incorrect. On the contrary if model selected correctly, the parameterised estimated PSDs are less biased and possess a lower variance than non parameterised estimated PSDs. Due to low computational complexity and good accuracy subspace methods have become very popular. Subspace-based methods rely on observations related to the Eigen decomposition of the complex exponents into a signal and a noise subspace [5].

\section{SUBSPACE ESTIMATION METHOD}

This method includes MUSIC and Eigen vector method for frequency estimation. Subspace method do not produce power estimates like power spectral density estimates and are most useful for the identification of frequency and sensitive to misspecification of model order.

MUSIC: This is an algorithm which is used for the estimation of frequency. It picks the peaks of DFT spectra in presence of noise and is able to estimate the frequencies with greater accuracy. In MUSIC method, entire noise subspace has signal vector superimposed onto it.. The calculation of eigenvector and Eigen values of the correlation matrix gives observation that smallest Eigen values of matrix corresponds to the noise subspace and largest corresponds to the signal subspace. In this method noise subspace is used to estimate the frequencies of sinusoidal components to form the superimposition and is given by:

Pnoise $=$ Enoise $E *$ noise $=I-$ Psignal

The pseudo spectrum of MUSIC is defined as: 


$$
\hat{P}\left(e^{j \omega}\right)=\left.\frac{1}{\sum_{i=p+1}^{M} E_{i}(z) E_{i}^{*}(z)}\right|_{z=e^{j \omega}}
$$

The denominator from (2) has double roots lying on the unit circle which gives the frequencies of the signal components and this method is thus called root-MUSIC. Now the powers of each component can be estimated from the eigen values and eigen vectors of the correlation matrix [6], using the relation:

$$
\mathbf{e}_{i}^{* T} \mathbf{R}_{x} \mathbf{e}_{i}=\lambda_{i}
$$

by substituting:

$$
\mathbf{R}_{x}=\sum_{i=1}^{p} \mathcal{E}\left\{A_{i} A_{i}^{*}\right\} \mathbf{s}_{i} \mathbf{s}_{i}^{T}+\sigma_{0}^{2} \mathbf{I}=\sum_{i=1}^{M} P_{i} s_{i} s_{i}^{{ }^{T} T}+\sigma_{0}^{2} \mathbf{I}
$$

where $P_{i}$-power of the $i^{\text {th }}$ component.

Eigen Vector: It is referred to as "super-resolution" method as it can resolve frequencies that are closely spaced. The problem that arises in spectral estimation is concerned with the removal of noise from uncorrelated sinusoids.

The signal model assume that the signal is given by

$$
x[n]=\sum_{i=1}^{p} a_{i} e^{j\left(n \omega_{i}+\phi_{i}\right)}
$$

Then

$$
r_{x x}[k]=E\{x[n] x[n-k]\}=\sum_{i=1}^{p} p_{i} e^{j n \omega_{i}}
$$

where

$$
p_{i}=a_{i}^{2}
$$

"The principal eigenvectors span the signal subspace". Thus given a sequence of observations we can determine the autocorrelation matrix and its eigenvectors [7]. Knowing the first eigenvectors we can develop the space in which the signals abode. Though, at this point we do not know their frequencies.

\section{BROADBAND \& NARROWBAND}

In communications, term band means bandwidth or range of frequencies. Its size is determined by $\mathrm{kHz}, \mathrm{MHz}, \mathrm{GHz}$ etc. In broadband communication as the name implies has broad range of frequency operation as and when compared to narrowband communication. Here, coherence bandwidth is exceeded which thus doesn't provides suppressed frequency response which means the gain is not constant throughout. Broadband communication can also be called as wideband communication. Wideband communication makes use of extremely short coded pulses which have time precision and is transmitted over a broad range of frequencies. In narrow band communication the gain remains constant for all frequencies in the range and thus provides flat frequency response as it doesn't exceed coherence bandwidth. Narrowband communication is achieved when the signal to be transmitted is superimposed by a sinusoidal carrier. The resultant signal possesses the sinusoidal nature and occupies a narrow band in the frequency domain [8]. In internet access broadband connections provides high date communication rate in comparison to narrowband connection. Modulation types that are used in narrowband communication are amplitude and angle (frequency and phase) modulation and in wideband system analog, digital and pulse position modulation which makes the use of optimum filters. Moreover broadband technologies have greater information carrying capacity with the same channel quality. A television may be considered as broadband as it receives wide range of channels and also used for streaming videos from internet with high bit rates.

\section{Resource of Radio Spectrum}

Radio spectrum is a finite resource in which sorting the efficient transmission of frequency becomes necessary because if the transmissions occurs at the same time in the same area and with the same frequency the interferences occurs. That is why it is important to incorporate tight radio regulations as most countries does for application of radio spectrum.

\section{Radio communication with extended application:}

Radio communication methods are broadly classified into FDMA, TDMA and CDMA. FDMA is the standard communication method that is broadly used for frequency below $1 \mathrm{GHz}$.Various kinds of high frequency radio modules and one-chip radio IC can be easily found making the component selection much easier for various applications.

\section{Suitable Frequency for Mobile Communication:}

The frequency band over $1 \mathrm{GHz}$ are used in Wireless LAN, Cband (satellite broadcast) and KU-band (fixed communication) and has property of stronger directionality transmission whereas the frequency band below $1 \mathrm{GHz}$ is well suited for mobile communication. High frequency radio wave has same properties as of light.

\section{Frequency available for licensing:}

In Europe, the $434 \mathrm{MHz}$ and $868 \mathrm{MHz}$ has been approved frequency band for ISM applications. Many more radio module and radio chip for these frequency bands have been provided by various manufacturers. Among them, the ICs based on direct conversion technologies are integrated with transceiver circuit and Phase locked loop synthesizer and Quadrature amplitude modulation/demodulation [9].

\section{Limited Transmission output power:}

Transmission of high power output is not allowed without the radio license. Generally, the minimum power limit is $5 \mathrm{~mW}$ and the maximum power limit is $10 \mathrm{~mW}$. Moreover, different countries have different power regulations. This limit is related to the product cost and application based on requested transmission range.

\section{RESULTS \& DISCUSSION}

PSD estimation with subspace method having MUSIC and Eigen vector which uses window in determining the quality of PSD has been shown in Fig. 1 \& Fig. 2. The main role of window is to damp out the effects of Gibbs phenomenon that results from truncation of an infinite series. It is a high resolution method that tends to produce better results. Here, rectangular window has been used with length $=4$ which has spectral leakage $(7.5 \%)$ and side lobe attenuation $(-11.3 \mathrm{~dB})$. Also, signal length is 32 with SNR $1 \mathrm{~dB}$ and estimation method has complex sinusoid $=2$. 


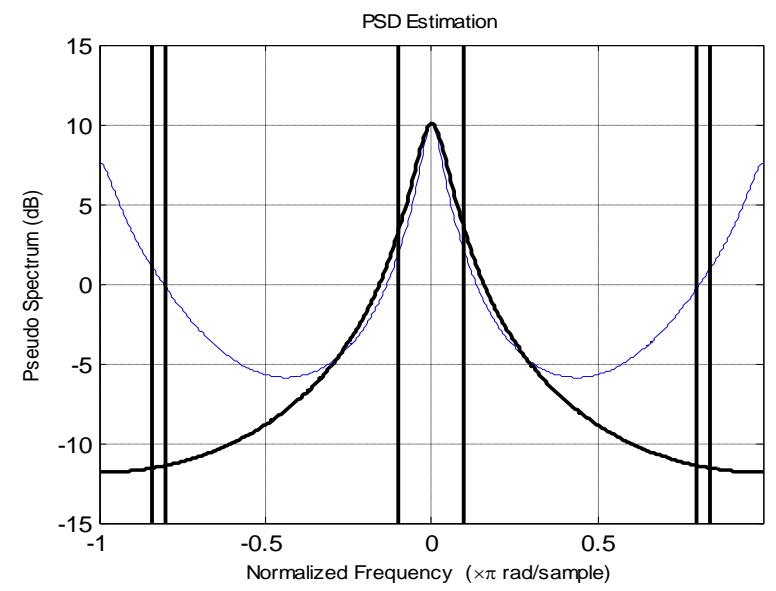

Figure 1- PSD estimation for MUSIC.

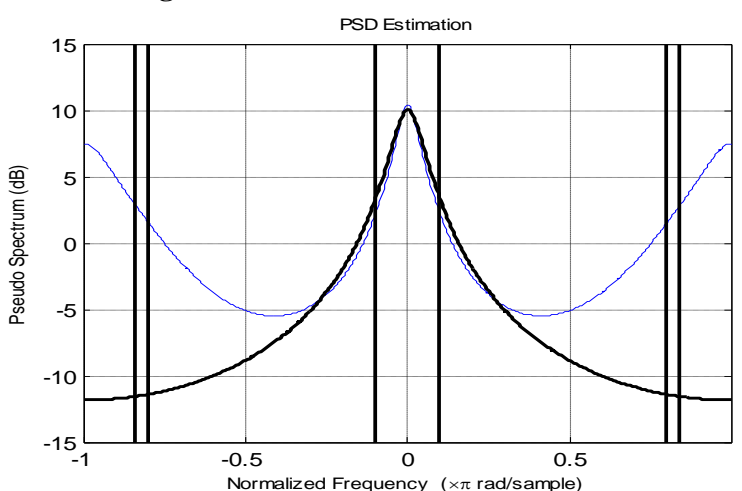

Figure 2- PSD estimation for Eigen Vector.

PSD estimation with parametric method including covariance and modified covariance has been shown in Fig. 3, Fig. 4 \& Fig. 5. In covariance estimation with $\mathrm{SNR}=1 \mathrm{~dB}$ the estimated PSD is not appropriately following the True PSD but when SNR is increased by $1 \mathrm{~dB}$ that means $\mathrm{SNR}=2 \mathrm{~dB}$ then it shows somehow better approximation. In modified covariance the $\mathrm{SNR}=1 \mathrm{~dB}$ shows estimation nearer to covariance with $\mathrm{SNR}=$ $2 \mathrm{~dB}$. Here, no windowing is being done and input signal has same length as that of subspace method.

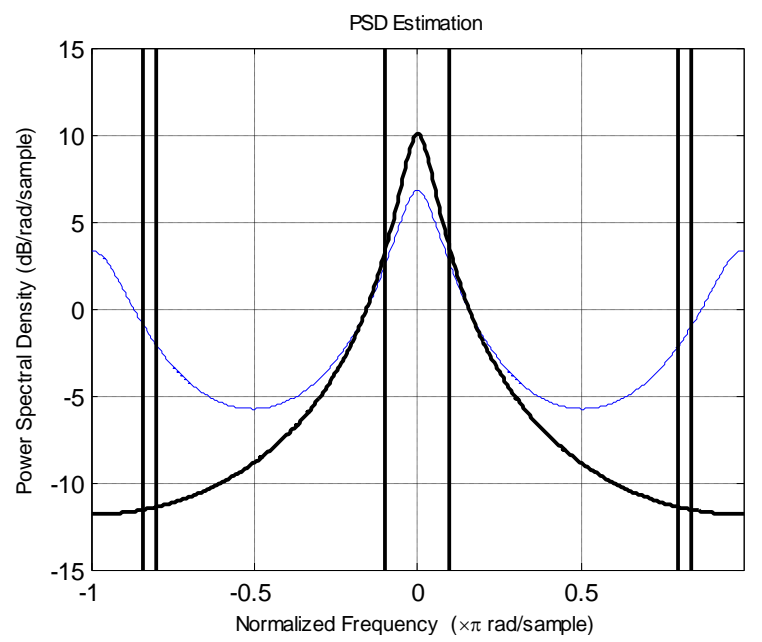

Figure 3-PSD estimation for Covariance with $\mathrm{SNR}=1 \mathrm{~dB}$.

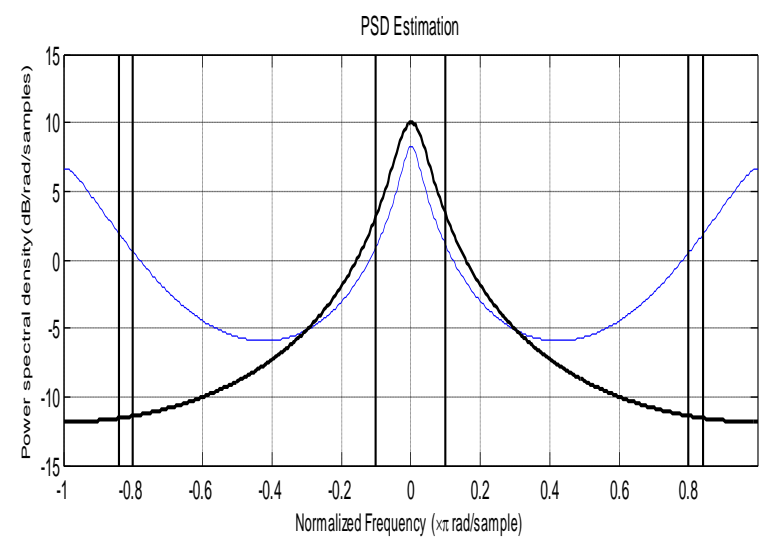

Figure 4- PSD estimation for Covariance with $\mathrm{SNR}=\mathbf{2 d B}$.

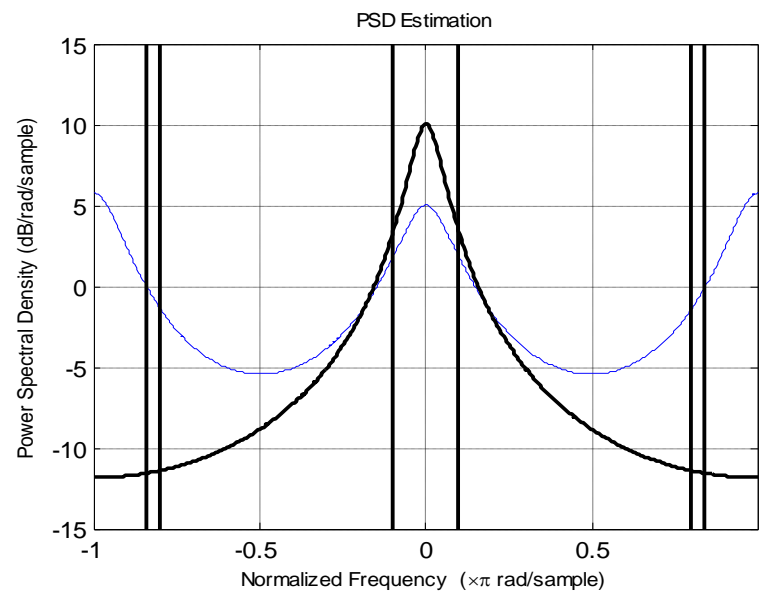

Figure 5- PSD estimation for Modified Covariance.

PSD estimation with non parametric method including Thompson Multitaper with no use of window and Modified Periodogram having $\mathrm{SNR}=1 \mathrm{~dB}$ with use of rectangular window having spectral leakage $(9.12 \%)$ and side lobe attenuation $(-13.2 \mathrm{~dB})$ has been shown in Fig. 6 \& Fig. 7. This method is not suitable as the noise is arising in both estimated PSD.

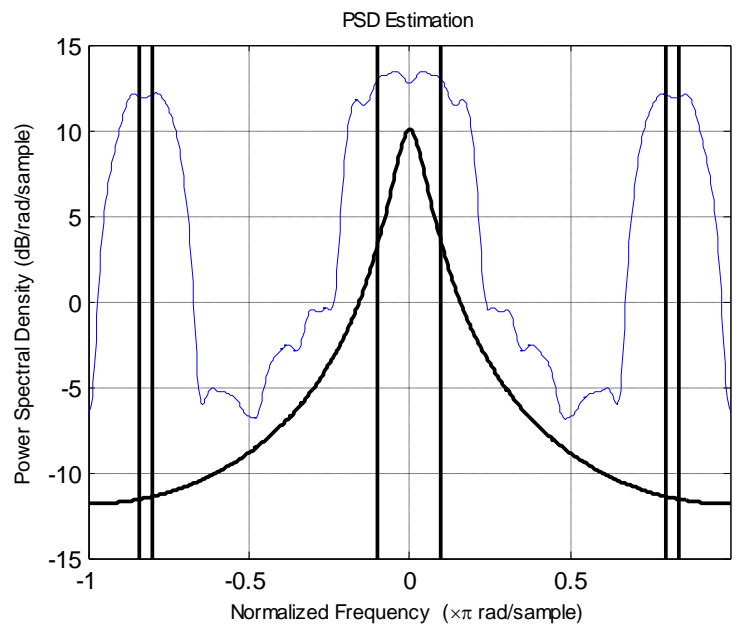

Figure 6- PSD estimation for Thompson Multitaper. 


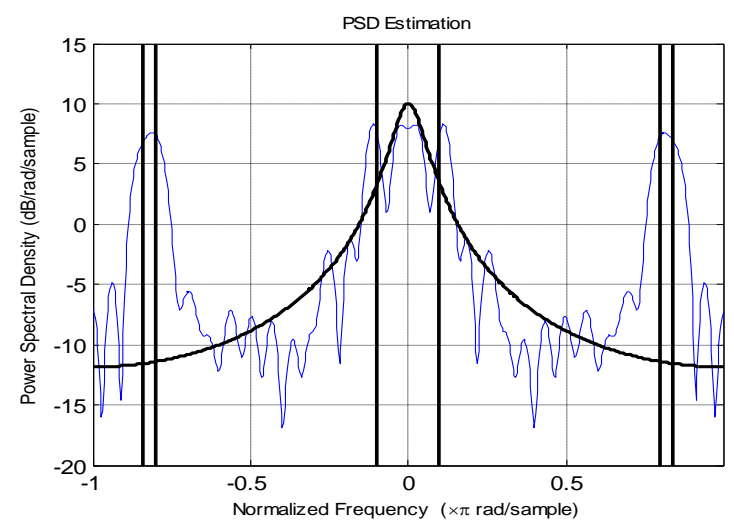

Figure 7- PSD estimation for Modified Periodogram.

\section{CONCLUSION}

Thus subspace power spectrum estimation gives appropriate result and is superior when compared to parametric and non parametric power spectrum estimation with estimated PSD nearer to true PSD. Subspace method is high resolution method which uses prior information of the spectral content of the signal to build a model of signal. That is why, subspace methods are more suitable for narrowband as well as broadband application as in non parametric, noise is arising in estimated PSD.

\section{REFERENCES}

[1] K Priya, R. Mehra, "Area Efficient Design of FIR Filter Using Symmetric Structure", International Journal of Advanced Research in Computer and Communication Engineering Vol. 1, Issue 10, pp. 842-845, 2012.

[2] Hansa Rani Gupta, Rajesh Mehra,"Power Spectrum Estimation using Welch method for various window techniques", International Journal of Scientific Research
Engineering and Technology, Vol 2, Issue 6, pp. 389392, 2013.

[3] Akanshya Shradhanjali, Subhra Chowdhury and Neelesh Kumar, " Power Spectral Density Estimation Of EMG signals Using Parametric and Non- Parametric Approach", Global Advanced Research Journal of Engineering Technology and Innovation, Vol. 2, Issue 4, pp. 111-117, 2013.

[4] P Kumar Rahi, Rajesh Mehra,"Änalysis of power spectrum estimation using Welch method for various window techniques", International Journal of Emerging Technologies and Engineering, Vol. 2, Issue 6 , pp.106109, 2014.

[5] Amit Kumar Chanchal, Rajesh Mehra,"Power spectrum estimation for band pass filter using non parametric methods", International Conference on Research in Electrical and Mechanical Engineering, pp.9-12, 2014.

[6] Jacek Rezmer. "Measurement of IEC Groups and Subgroups using Advanced Spectrum Estimation Methods", IEEE Instrumentation and Measurement Technology Conference Proceedings, Vol 57, Issue 4, pp. 672-681, 2008

[7] S. Ejaz and M. A. Shafiq, "Comparison of spectral and subspace algorithim for FM source estimation", Progress In Electromagnetics Research, Vol. 14, pp.11-21, 2010.

[8] Vivek Akarte, Nitin Punse and Ankush Dhanorka “ Power Line Communication System”, International Journal of Innovative Research in Electrical, Electronics, Instrumentation and Control Engineering Vol. 2, Issue 1,pp. 709-713, 2014.

[9] Yukinaga Koike, Circuit Design Inc, "Difference between Wide band and Narrow band Radio Module, pp.1, 2002. 